\title{
Pericytes from Brain Microvessels Strengthen the Barrier Integrity in Primary Cultures of Rat Brain Endothelial Cells
}

\author{
Shinsuke Nakagawa $\cdot$ Mária A. Deli $\cdot$ Shinobu Nakao · \\ Masaru Honda · Kentaro Hayashi · Ryota Nakaoke • \\ Yasufumi Kataoka $\cdot$ Masami Niwa
}

Received: 3 August 2007/ Accepted: 5 August 2007 / Published online: 6 September 2007

(C) Springer Science+Business Media, LLC 2007

\begin{abstract}
The blood-brain barrier (BBB) characteristics of cerebral endothelial cells are induced by organ-specific local signals. Brain endothelial cells lose their phenotype in cultures without cross-talk with neighboring cells. (2) In contrast to astrocytes, pericytes, another neighboring cell of endothelial cells in brain capillaries, are rarely used in BBB co-culture systems. (3) Seven different types of BBB models, mono-culture, double and triple co-cultures, were constructed from primary rat brain endothelial cells, astrocytes and pericytes on culture inserts. The barrier integrity of the models were compared by measurement of transendothelial electrical resistance and permeability for the small molecular weight marker fluorescein. (4) We could confirm that brain endothelial monolayers in mono-culture do not form tight barrier. Pericytes induced higher electrical resistance and lower permeability for fluorescein than type I astrocytes in co-culture conditions. In triple co-culture models the tightest barrier was observed when endothelial cells and pericytes were positioned on the two sides of the
\end{abstract}

S. Nakagawa $\cdot$ M. A. Deli $\cdot$ S. Nakao $\cdot$ R. Nakaoke $\cdot$ M. Niwa

Department of Pharmacology, Nagasaki University Graduate School of Biomedical Sciences,

1-12-4 Sakamoto, Nagasaki 852-8523, Japan

S. Nakagawa $\cdot$ M. A. Deli $(\bowtie) \cdot$ Y. Kataoka $\cdot$ M. Niwa

BBB Laboratory, PharmaCo-Cell Co. Ltd, 1-12-4 Sakamoto, Nagasaki 852-8523, Japan

e-mail: deli@pharmacocell.co.jp

M. A. Deli

Laboratory of Molecular Neurobiology, Institute of Biophysics, Biological Research Center, Temesvári körút 62, Szeged H-6726, Hungary

M. Honda $\cdot$ K. Hayashi

Department of Neurosurgery, Nagasaki University Graduate School of Biomedical Sciences, 1-12-4 Sakamoto, Nagasaki 852-8523, Japan

Y. Kataoka

Department of Pharmaceutical Care and Health Sciences, Faculty of Pharmaceutical Sciences, Fukuoka University, 8-19-1 Nanakuma, Fukuoka 814-0180, Japan 
porous filter membrane of the inserts and astrocytes at the bottom of the culture dish. (5) For the first time a rat primary culture based syngeneic triple co-culture BBB model has been constructed using brain pericytes beside brain endothelial cells and astrocytes. This model, mimicking closely the anatomical position of the cells at the BBB in vivo, was superior to the other BBB models tested. (6) The influence of pericytes on the BBB properties of brain endothelial cells may be as important as that of astrocytes and could be exploited in the construction of better BBB models.

Keywords Pericytes - Blood-brain barrier - Brain endothelial cells ·

Astrocytes · Co-culture - In vitro BBB model (rat) .

Permeability · Transendothelial electrical resistance

\section{Introduction}

The endothelial cells in the blood vessels of different organs possess different characteristics, regulated by specific local signals. Brain capillary endothelial cells forming the anatomical basis of the blood-brain barrier (BBB) show both endothelial and epithelial features (Joó 1996). Cells surrounding brain capillaries, such as astrocytes, pericytes, perivascular microglia, and neurons contribute to the formation and maintenance of a functional $\mathrm{BBB}$ in the central nervous system. Among these cells, astrocytes were the first to be recognized as regulators of brain endothelial characteristics and functions (for reviews see Abbott 2005; Abbott et al. 2006; Haseloff et al. 2005) including the induction of tight junctions (TJs) (Tao-Cheng et al. 1987), a fundamental characteristic of the BBB. Brain pericytes, the cells sharing the basal membrane with capillary endothelial cells, were recently found to be also able to induce BBB functions in cultured brain endothelial cells (Hayashi et al. 2004; Dohgu et al. 2005). Despite their localization as the nearest neighbors of endothelial cells with a fundamental role in stabilizing brain capillary structure in vivo (Hellström et al. 2001), few data are available on the functional significance of pericytes on BBB properties.

The discovery of cell-culture inserts with porous filter membranes allowed the use of brain endothelial cells for permeability studies in vitro. Since then it has definitely been shown by accumulating evidences that the technique of co-culture of BBB-related cells using the setup is a suitable in vitro reconstituted BBB model to clarify cross-talk between the endothelial cells and cells regulating BBB functions (Deli et al. 2005). The transendothelial electrical resistance (TEER) and transendothelial permeability coefficient $\left(\mathrm{P}_{\mathrm{e}}\right)$ for small water-soluble inert tracers are straightforward methods to evaluate TJ function and BBB paracellular transport, two important paremeters of the quality of BBB models (Deli et al. 2005).

Our aim was to establish in vitro BBB models using rat brain capillary endothelial cells, pericytes, and astrocytes and to compare the barrier tightness of these systems. For this purpose, mono-cultures, double and triple co-culture models were constructed and their BBB functions were evaluated by measuring TEER and $\mathrm{P}_{\mathrm{e}}$. With these experiments we also wanted to clarify the participation of pericytes in the induction of BBB properties. 


\section{Materials and Methods}

All reagents were purchased from Sigma, USA, unless otherwise indicated. Wistar rats were obtained from Japan SLC Inc., Japan. All animals were treated in strict accordance with the NIH Guide for Care and Use of Laboratory Animals (NIH Publications No. 80-23) and as approved by the Nagasaki University Animal Care Committee.

\section{Cell Cultures}

Primary cultures of rat brain capillary endothelial cells (RBEC) were prepared from 3-week-old rats, as previously described (Deli et al. 1997). Meninges were carefully removed from forebrains and gray matter was minced into small pieces of approximately $1 \mathrm{~mm}^{3}$ in ice-cold Dulbecco's modified Eagle's medium (DMEM), then dissociated by 25 times of up- and down-strokes with a 5-ml pipette in DMEM containing collagenase type $2(1 \mathrm{mg} / \mathrm{ml}$, Worthington, USA), $300 \mu \mathrm{l}$ DNase $(15 \mu \mathrm{g} / \mathrm{ml})$, gentamicin $(50 \mu \mathrm{g} / \mathrm{ml})$, and then digested in a shaker for $1.5 \mathrm{~h}$ at $37^{\circ} \mathrm{C}$. The cell pellet was separated by centrifugation in $20 \%$ bovine serum albumin (BSA)-DMEM $(1,000 \mathrm{~g}$, $20 \mathrm{~min})$. The microvessels obtained in the pellet were further digested with collagenasedispase $\left(1 \mathrm{mg} / \mathrm{ml}\right.$, Roche, Switzerland) and DNase $\left(6.7 \mu \mathrm{g} / \mathrm{ml}\right.$ in DMEM for $1 \mathrm{~h}$ at $37^{\circ} \mathrm{C}$. Microvessel endothelial cell clusters were separated on a $33 \%$ continuous Percoll (Pharmacia, Sweden) gradient, collected and washed twice in DMEM before plating on $35-\mathrm{mm}$ plastic dishes coated with collagen type IV and fibronectin (both $0.1 \mathrm{mg} / \mathrm{ml}$ ). RBEC cultures were maintained in DMEM/F12 supplemented with $10 \%$ plasma derived serum (PDS, Animal Technologies, USA), basic fibroblast growth factor (bFGF, Roche, Switzerland, $1.5 \mathrm{ng} / \mathrm{ml})$, heparin $(100 \mu \mathrm{g} / \mathrm{ml})$, insulin $(5 \mu \mathrm{g} / \mathrm{ml})$, transferrin $(5 \mu \mathrm{g} / \mathrm{ml})$, sodium selenite $(5 \mathrm{ng} / \mathrm{ml})$ (insulin-transferrin-sodium selenite media supplement), gentamicin $(50 \mu \mathrm{g} / \mathrm{ml})$, and puromycin $(4 \mu \mathrm{g} / \mathrm{ml})$ (Perrière et al. 2005) (RBEC medium I) at $37^{\circ} \mathrm{C}$ with a humidified atmosphere of $5 \% \mathrm{CO}_{2} / 95 \%$ air, for 2 days. On the third day, the cells received a new medium which contained all the components of RBEC medium I except puromycin (RBEC medium II). When the cultures reached $80 \%$ confluency (fourth day in vitro), the purified endothelial cells were passaged by a brief treatment with trypsin $(0.05 \% \mathrm{wt} / \mathrm{vol})$-EDTA $(0.02 \% \mathrm{wt} / \mathrm{vol})$ solution, and used to construct various types of in vitro BBB models.

Rat cerebral astrocytes were obtained from neonatal Wistar rats. Meninges were removed and cortical pieces mechanically dissociated in astrocyte culture medium (DMEM supplemented with $10 \%$ fetal bovine serum). Dissociated cells were seeded into cell culture flasks. In order to obtain type 1 astrocytes, flasks with confluent cultures were shaken at $37^{\circ} \mathrm{C}$ overnight. The purity of astrocytes was checked by immunostaining for glial fibrillary acidic protein (GFAP), and the cells were used at passage 2 .

Pure cultures of rat cerebral pericytes were obtained by a prolonged, 2-week culture of isolated brain microvessel fragments, that contain pericytes beside endothelial cells. The same preparations yield primary RBEC after puromycin-treatment. Pericyte survival and proliferation was favored by selective culture conditions, using uncoated dishes, and DMEM supplemented with $10 \%$ fetal bovine serum and antibiotics. Culture medium was changed every 3 days. Pericytes were characterized by their large size and branched morphology, positive immunostaining for $\alpha$-smooth muscle actin, and absence 
of von Willebrand factor and GFAP staining. Pericytes and astrocytes were frozen in cryo-medium CELLBANKER ${ }^{\circledR}$ (BCL-1, ZENOAQ, Japan), and stored at $-80^{\circ} \mathrm{C}$.

\section{Construction of In vitro BBB models}

The day when the endothelial cells were plated and models were established was defined as day zero in vitro (Day 0). To construct various in vitro models of BBB, pericytes or astrocytes $\left(1.5 \times 10^{4}\right.$ cells $\left./ \mathrm{cm}^{2}\right)$ were seeded on the bottom side of the collagen-coated polyester membrane of the Transwell ${ }^{\circledR}$ inserts. The cells were let to adhere firmly for overnight, then endothelial cells $\left(1.5 \times 10^{5}\right.$ cells $\left./ \mathrm{cm}^{2}\right)$ were seeded on the upper side of the inserts placed in the well of the 12-well culture plates containing no cells, pericytes, or astrocytes (out-of-contact types, Fig. 1). BBB models were maintained in RBEC medium II. From Day 1, the culture medium was supplemented with $500 \mathrm{nM}$ hydrocortisone (Hoheisel et al. 1998). Under these conditions, in vitro BBB models were established within 3 days after setting of the cells. Seven types of BBB models were constructed as shown on the scheme (Fig. 1). As negative controls, astrocytes and pericytes, which do not form barrier, were cultured on the inserts, respectively.

\section{Evaluation of the Barrier Integrity}

TEER, which reflects in culture conditions the flux of mainly sodium ions through cell layers, was measured by Epithelial-volt-ohm meter and Endohm-12 chamber electrodes (World Precision Instruments, USA). TEER of coated, but cell-free filters was subtracted from measured TEER values of the models shown as $\Omega \times \mathrm{cm}^{2}$.

The flux of sodium fluorescein (Na-F) across endothelial monolayers was determined as previously described (Kis et al. 2001). Cell culture inserts were transferred to 12-well plates containing $1.5 \mathrm{ml}$ assay buffer $\left(136 \mathrm{mM} \mathrm{NaCl}, 0.9 \mathrm{mM} \mathrm{CaCl}_{2}, 0.5 \mathrm{mM} \mathrm{MgCl}\right.$, $2.7 \mathrm{mM} \mathrm{KCl}, 1.5 \mathrm{mM} \mathrm{KH} \mathrm{PO}_{4}, 10 \mathrm{mM} \mathrm{NaH} \mathrm{PO}_{4}, 25 \mathrm{mM}$ glucose, and $10 \mathrm{mM}$ Hepes,

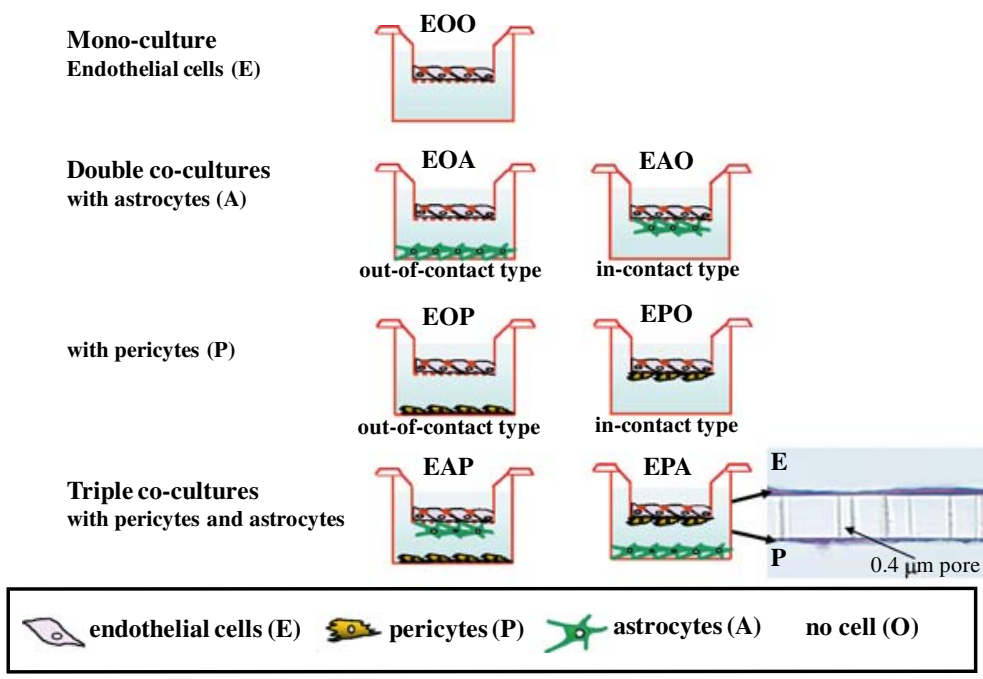

Fig. 1 A scheme of BBB models 
$\mathrm{pH}$ 7.4) in the basolateral or lower compartments. In the inserts culture medium was replaced by $0.5 \mathrm{ml}$ buffer containing $10 \mathrm{mg} / \mathrm{ml} \mathrm{Na-F}$ (MW: $376 \mathrm{Da}$ ). The inserts were transferred at 5, 15, and $30 \mathrm{~min}$ to a new well containing assay buffer. The concentrations of the marker molecule in samples from the upper and lower compartments were determined by fluorescence multiwell plate reader (excitation: $485 \mathrm{~nm}$, emission: $535 \mathrm{~nm}$ ). Flux across cell-free inserts was also measured. Transport was expressed as $\mu \mathrm{l}$ of donor (luminal) compartment volume from which the tracer is completely cleared. Transendothelial permeability coefficient $\left(\mathrm{P}_{\mathrm{e}}\right.$ in $\left.10^{-6} \mathrm{~cm} / \mathrm{s}\right)$ was calculated as previously described (Deli et al. 2005).

All data presented are means \pm SEM. The values were compared using the analysis of variance followed by Bonferroni-Dunn test. Changes were considered statistically significant at $P<0.05$. All experiments were repeated at least three times, and the number of parallel inserts was four.

\section{Results and Discussion}

As it was demonstrated by several groups before (Deli et al. 2005), co-culture of RBEC with astrocytes either in contact (EA0) or out of contact (E0A) arrangement could significantly increase the tightness of endothelial monolayers. From the two models, the in contact type EA0 was more effective in enhancing the barrier properties. The presence of pericytes (EP0 and E0P) elevated the TEER of RBEC by about $400 \%$ at Day 5 (Fig. 2), an effect that was significantly higher $(P<0.05$ and 0.01$)$, than that of astrocytes, standardly used in BBB models. Astrocytes or pericytes do not form any barrier, as reflected by their very low resistance (Fig. 2 inset). The TEER values of the triple co-culture models were even higher than that of the double co-culture models. The level of TEER in EPA type increased up to $388 \pm 18.8 \Omega \times \mathrm{cm}^{2}$ at Day 5 (Fig. 2), the highest value of the seven models tested, and significantly differing from all other

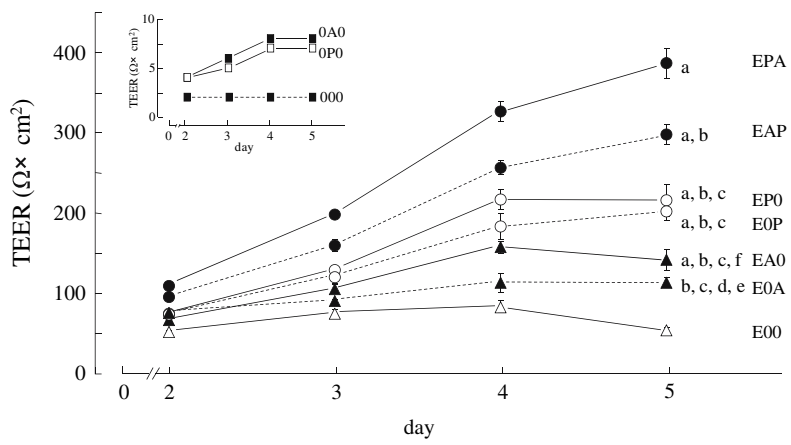

Fig. 2 Effect of co-culture on the induction of transendothelial electrical resistance (TEER) in brain capillary endothelial cell monolayers of in vitro BBB models, E00 (open triangles), E0A (filled triangles, dotted line), EA0 (filled triangles), E0P (open circles, dotted line), EP0 (open circles), EAP (filled circles, dotted line), EPA (filled circles). An inset graph (left upper panel) indicates a very low TEER of astrocytes (filled square, 0A0) and pericytes (open square, 0P0) cultured on the inserts. Results $\left(\Omega \times \mathrm{cm}^{2}\right)$ are presented as means \pm SEM. $n=4$. ${ }^{a} P<0.01$ was considered statistically significant difference in the TEER, as compared to E00, ${ }^{\mathrm{b}} P<0.01$ to EPA, ${ }^{\mathrm{c}} P<0.01$ to EAP, ${ }^{\mathrm{d}} P<0.01$ to EP0, ${ }^{\mathrm{e}} P<0.01$ to E0P, and ${ }^{\mathrm{f}} P<0.05$ to EP0, respectively 
models. High TEER was observed in the models constructed with pericytes, as compared with those without pericytes. In addition, endothelial cells in contact with pericytes (EP0, EPA) showed higher TEER than those out of contact with pericytes (E0P, EAP).

The paracellular permeability of RBEC monolayers measured by the water soluble small marker fluorescein was the highest in endothelial mono-cultures, indicating the leakiest barrier (Fig. 3). The presence of astrocytes or pericytes in either double or triple co-culture systems significantly decreased the flux of the tracer. In double co-culture models the in-contact types EA0 and EP0 showed lower permeability for Na-F than the non-contact types E0A and E0P (Fig. 3).

Our results on pericytic induction of BBB properties are in agreement with recent publications. Rat pericytes could decrease the paracellular permeability and enhance $P$-glycoprotein activity in a mouse brain endothelial cell line, MBEC4 (Dohgu et al. 2005). The mRNA expression of MRP6 efflux pump was also up-regulated in bovine brain endothelial cells by pericytes (Berezowski et al. 2004). Some of the factors mediating this effect have been identified. The upregulation of BBB properties in MBEC4 cells was induced by transforming growth factor- $\beta$ production in pericytes (Dohgu et al. 2005). Pericyte-derived angiopoietin-1 enhanced occludin gene expression in a brain endothelial cell line (Hori et al. 2004). Moreover, extracellular matrix from pericytes improved the tightness of porcine cerebral endothelial cells (Hartmann et al. 2007).

The triple BBB models published earlier used combinations of brain endothelial cells, astrocytes and neurons. The presence of both astrocytes and neurons decreased the paracellular permeability of RBE4 immortalized rat brain endothelial cells (Schiera et al. 2005). In a flow-based in vitro BBB model, the differentiation of serotonergic neurons was promoted by the endothelial-glial co-culture (Stanness et al. 1999). Although EPA is not the first BBB model using three cell types, this is the first rat primary culture-based syngeneic model that uses brain pericytes.

These results clearly confirm that cellular communications play an important role in inducing and maintaining the barrier function of brain endothelial cells. Brain endothelial cells without the influence of astrocytes or pericytes showed the weakest barrier tightness, indicating the loss of BBB properties in culture conditions in the absence of cellular cross-talk.

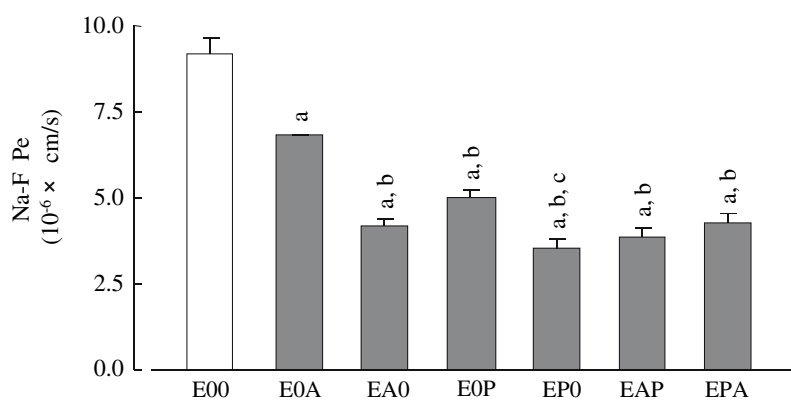

Fig. 3 Transendothelial permeability $(\mathrm{Pe})$ changes for paracellular permeability marker sodium fluorescein $(\mathrm{Na}-\mathrm{F})$ in brain capillary endothelial cell monolayers of in vitro BBB models. Results $\left(10^{-6} \mathrm{~cm} / \mathrm{s}\right)$ are presented as means \pm SEM. $n=4 .{ }^{\mathrm{a}} P<0.01$ was considered statistically significant difference in the $\mathrm{P}_{\mathrm{e}}$, as compared to E00, ${ }^{\mathrm{b}} \mathrm{P}<0.01$ to E0A, and ${ }^{\mathrm{c}} \mathrm{P}<0.05$ to E0P, respectively 
The major finding of the experiments is that brain microvessel pericytes are able to strengthen the BBB function of endothelial cells, like astrocytes, widely used in BBB models. Pericytes could induce a tighter barrier than astrocytes. The in-contact models were tighter than the out-of-contact types, and triple models showed better barrier properties than double co-cultures. As brain endothelial cells, pericytes and astrocytes, composing the brain microvasculature, have their own roles at the BBB, the EPA co-culture system constructed with all three types of cells seems to be a promising new in vitro BBB model.

Acknowledgments The work was partly supported by Grants for New Industry Creative-Type Technology R\&D promotion Programs from the Ministry of Economy, Trade and Industry (2005, 2006), Grant-In-Aid for Scientific Research from the Ministry Education, Culture, Sports, Science and Technology, Japan, and Grants from the Hungarian Research Fund (OTKA T37834) and National Office for Research and Technology (RET 08/2004).

\section{References}

Abbott NJ (2005) Dynamics of CNS barriers: evolution, differentiation, and modulation. Cell Mol Neurobiol 25:5-23

Abbott NJ, Ronnback L, Hannson E (2006) Astrocyte-endothelial interactions at the blood-brain barrier. Nat Rev Neurosci 7:41-53

Berezowski V, Landry C, Dehouck M-P, Cecchelli R, Fenart L (2004) Contribution of glial cells and pericytes to the mRNA profiles of P-glycoprotein and multidrug resistance-associated proteins in an in vitro model of the blood-brain barrier. Brain Res 1018:1-9

Deli MA, Szabó CA, Dung NTK, Joó F (1997) Immunohistochemical and electron microscopy detections on primary cultures of rat cerebral endothelial cells. In: Boer AG, Sutanto W (eds) Drug transport across the blood-brain barrier: in vivo and in vitro techniques. Harwood Academic Publishers, Amsterdam, pp 23-28

Deli MA, Ábrahám CS, Kataoka Y, Niwa M (2005) Permeability studies on in vitro blood-brain barrier models: physiology, pathology, and pharmacology. Cell Mol Neurobiol 25:59-127

Dohgu S, Takata F, Yamauchi A, Nakagawa S, Egawa T, Naito M, Tsuruo T, Sawada Y, Niwa M, Kataoka Y (2005) Brain pericytes contribute to the induction and up-regulation of blood-brain barrier functions through transforming growth factor-beta production. Brain Res 1038:208-215

Hartmann C, Zozulya A, Wegener J, Galla HJ (2007) The impact of glia-derived extracellular matrices on the barrier function of cerebral endothelial cells: an in vitro study. Exp Cell Res 313:1318-1325

Haseloff RF, Blasig IE, Bauer H-C, Bauer H (2005). In search of the astrocytic factor(s) modulating blood-brain barrier functions in brain capillary endothelial cells in vitro. Cell Mol Neurobiol 25:2539

Hayashi K, Nakao S, Nakaoke R, Nakagawa S, Kitagawa N, Niwa M (2004) Effects of hypoxia on endothelial/pericytic co-culture model of the blood-brain barrier. Regul Pept 123:77-83

Hellström M, Gerhardt H, Kalén M, Li X, Eriksson U, Wolburg H, Betsholtz C (2001) Lack of pericytes leads to endothelial hyperplasia and abnormal vascular morphogenesis. J Cell Biol 153:543-553

Hoheisel D, Nitz T, Franke H, Wegener J, Hakvoort A, Tilling T, Galla HJ (1998) Hydrocortisone reinforces the blood-brain barrier properties in a serum free cell culture system. Biochem Biophys Res Commun 247:312-315

Hori S, Ohtsuki S, Hosoya K, Nakashima E, Terasaki T (2004) A pericyte-derived angiopoietin-1 multimeric complex induces occludin gene expression in brain capillary endothelial cells through Tie-2 activation in vitro. $\mathrm{J}$ Neurochem 89:503-513

Joó F (1996) Endothelial cells of the brain and other organ systems: some similarities and differences. Prog Neurobiol 48:255-273

Kis B, Deli MA, Kobayashi H, Ábrahám CS, Yanagita T, Kaiya H, Isse T, Nishi R, Gotoh S, Kangawa K, Wada A, Greenwood J, Niwa M, Yamashita H, Ueta Y (2001) Adrenomedullin regulates bloodbrain barrier functions in vitro. Neuroreport 12:4139-4142

Perrière N, Demeuse P, Garcia E, Regina A, Debray M, Andreux J-P, Couvreur P, Schermann J-M, Temsamani J, Couraud P-O, Deli MA, Roux F (2005) Puromycin-based purification of rat brain capillary endothelial cell cultures. Effect on the expression of blood-brain barrier-specific properties. J Neurochem 93:279-289 
Schiera G, Sala S, Gallo A, Raffa MP, Pitarresi GL, Savettieri G, Di Liegro I (2005) Permeability properties of a three-cell type in vitro model of blood-brain barrier. J Cell Mol Med 9:373-379

Stanness KA, Neumaier JF, Sexton TJ, Grant GA, Emmi A, Maris DO, Janigro D (1999) A new model of the blood-brain barrier: co-culture of neuronal, endothelial and glial cells under dynamic conditions. Neuroreport 10:3725-3731

Tao-Cheng JH, Nagy Z, Brightman MW (1987) Tight junctions of brain endothelium in vitro are enhanced by astroglia. J Neurosci 7:3293-3299 